\title{
Consumo de sustancias psicoactivas en pacientes con trastornos psicóticos ingresados en Sala de Emergencia psiquiátrica. Hospital Vilardebó. Montevideo. Uruguay Psychoactive substance use in patients with psychotic disorders admitted to psychiatric emergency room. Hospital Vilardebó. Montevideo. Uruguay
}

Pablo Fielitz G. ${ }^{1}$, Héctor Suárez C. ${ }^{2}$, Marcelo Escobal L. ${ }^{3}$, María Alcira Frontini L. ${ }^{3}$, Gabriela López-Rega ${ }^{3}$, Viviana Navarro T. ${ }^{4}$, Luis Rodríguez-Formoso ${ }^{4}$, Andrea Storch O. ${ }^{4}$, Soledad García D. ${ }^{5}$ Y Osvaldo do Campo C. ${ }^{6}$

A descriptive study was conducted from October to December 2008 in the emergency room of Hospital Vilardebó (State Mental Facility) to establish the prevalence of psychoactive substance use in patients admitted for Psychotic Disorders. The presence of recent use of substance was established by self-report and urine test for marijuana and cocaine / cocaine base. The prevalence of recent use in patients with psychotic disorders was $17.9 \%$. Patients who tested positive showed a total hospitalization rate greater than that of the group of nonusers, and hospitalization in the last year were clearly higher for the group who uses cocaine and its derivatives.

Key words: Psychoactive substances, psychosis, Emergency Room, Psychiatric Hospital. Rev Chil Neuro-Psiquiat 2010; 48 (2): 106-113

\section{Introducción}

El Hospital Vilardebó es el mayor Hospital Psiquiátrico de Agudos del país, contando con 330 camas de internación, dependiendo del Ministerio de Salud Pública.

El número de hospitalizaciones anuales para el año 2008 fue de 1.926, siendo la mayoría de ellas re-hospitalizaciones (aproximadamente dos tercios tienen uno o más ingresos anteriores en ese hospital). El promedio de ingresos mensuales fue de 161 para el año 2008, y aproximadamente el 44\% de ellos correspondieron a Psicosis ${ }^{1}$ (Código F2x de la CIE 10).

Recibido: 9/12/2009

Aceptado: 11/04/2010

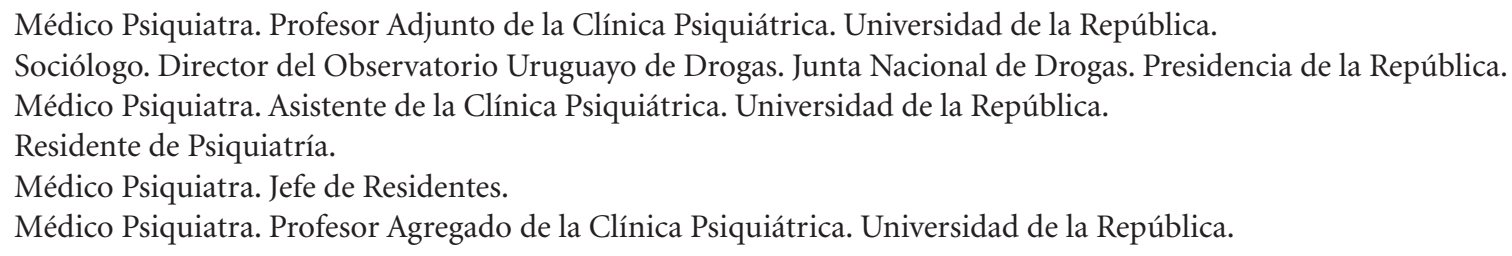


De acuerdo a los censos de población internada en este hospital en los años 2005, 2006 y 2007, el diagnóstico de Dependencia de polisustancias fue el primer diagnóstico de co-morbilidad más frecuente $^{1}$ (F 19 CIE 10).

A los efectos del presente estudio definimos como "patología dual" a la existencia simultánea de un Trastorno por Dependencia o Abuso de Sustancias y un Trastorno psicótico. La presencia de co-morbilidad en esta área supera el $60 \%{ }^{2,3}$. Se piensa que alrededor de la mitad de todos los esquizofrénicos abusan de drogas o alcohol ${ }^{4}$. A su vez el consumo de Pasta Base de Cocaína podría provocar recidiva de psicosis 5 .

En el presente estudio se aborda la prevalencia del consumo de sustancias en el universo de los pacientes con trastornos psicóticos que acuden a Emergencia en situaciones que ameritan internación.

\section{Objetivos del presente estudio}

1. Establecer la prevalencia del consumo reciente de sustancias psicoactivas entre los pacientes con trastornos psicóticos que ingresaron al Hospital Vilardebó en el período de estudio.

2. Comparar la tasa de reingresos de los pacientes con consumo reciente $v s$ aquellos con testado negativo.

\section{Materiales y Métodos}

El estudio es descriptivo y se llevó a cabo desde el mes de octubre hasta diciembre del año 2008 en el Servicio de Emergencia del Hospital Vilardebó.

Se relevaron datos socio-demográficos (sexo, edad, ocupación, barrio de residencia), Motivo de Ingreso, antecedentes personales psiquiátricos (diagnóstico actual, diagnósticos anteriores, número total de internaciones previas, número de internaciones en el último año), patrón de consumo de diversas sustancias psicoactivas (Figura 1).

Dichos datos se obtuvieron tanto de la entrevista con el paciente como de la historia clínica.

Los pacientes que ingresaron al estudio son aquellos que cumplieron con los criterios de inclusión, es decir que habiendo consultado en
Emergencia ingresaron para internación en sala de observación o sala general con diagnóstico de trastorno psicótico realizado por el Médico Psiquiatra de guardia de acuerdo con la Clasificación Internacional de Enfermedades de la Organización Mundial de la Salud CIE 10: Esquizofrenia (F20), Trastornos Psicóticos Agudos y Transitorios (F23), Trastorno de Ideas Delirantes Persistentes (F22) o Psicosis Inducida por Sustancias (F1x.5). Se excluyeron los diagnósticos de Trastornos del Humor.

Se aplicó un formulario prediseñado basado en un prototipo del Sistema Interamericano de Datos Unificados sobre Consumo de Drogas (SIDUC, CICAD, OPS) al que se anexó información referente a la historia psiquiátrica del paciente (Figura 1).

La recolección de datos fue realizada por personal especializado y debidamente entrenado (Médicos Residentes y Postgrados de Psiquiatría, Docentes de Psiquiatría).

Previo a la aplicación del cuestionario anónimo se obtuvo consentimiento informado del familiar o Director del Hospital.

Se realizó recolección de muestra de orina para la detección de metabolitos de marihuana y cocaína en las primeras veinticuatro horas de consulta. Para dicha detección se utilizaron tiras reactivas dobles para Marihuana (THC)/Cocaína (COC) "DiagnoTest", procedencia República Popular China, Registro MSP 27978, Lot DOA 7010011, Certificado MSP $\mathrm{N}^{\circ}$ 502, con límite de corte para cocaína de $300 \mathrm{ng} / \mathrm{ml}$ y $50 \mathrm{ng} / \mathrm{ml}$ para Marihuana. Las mismas fueron donadas por Laboratorios Celsius. Las tiras reactivas detectan la presencia de cocaína independientemente de la vía de administración o la presentación (clorhidrato o pasta base de cocaína (PBC)).

Los datos se ingresaron al programa Statistical Package for the Social Sciences versión 16 (SPSS v16) a los efectos de ser procesados.

Para el análisis estadístico de los datos se realizó test de chi cuadrado y test de Anova.

\section{Resultados}

Se realizaron en total 194 registros que corresponden a 181 pacientes. La diferencia se atribuye a 


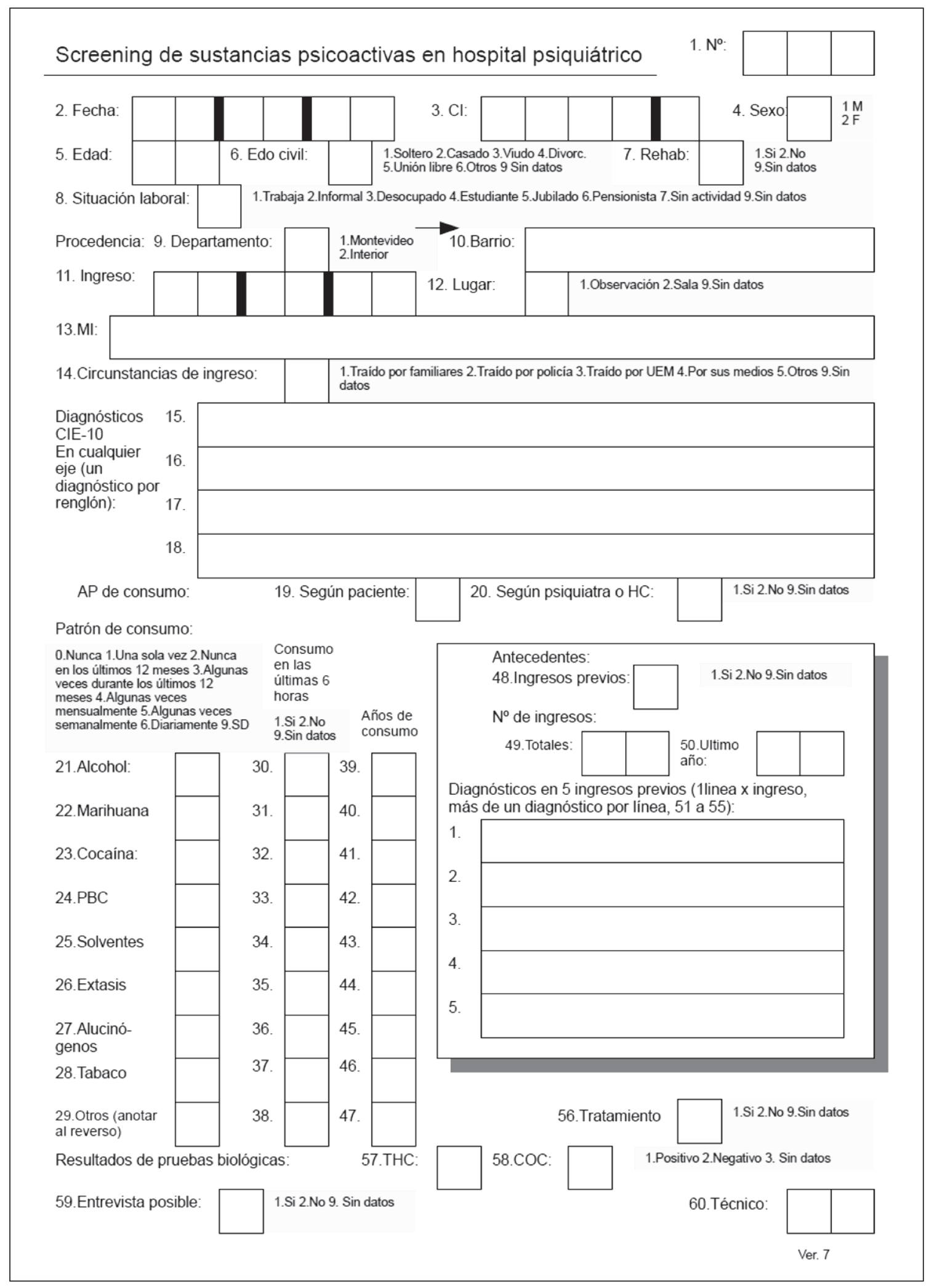

Figura 1. 


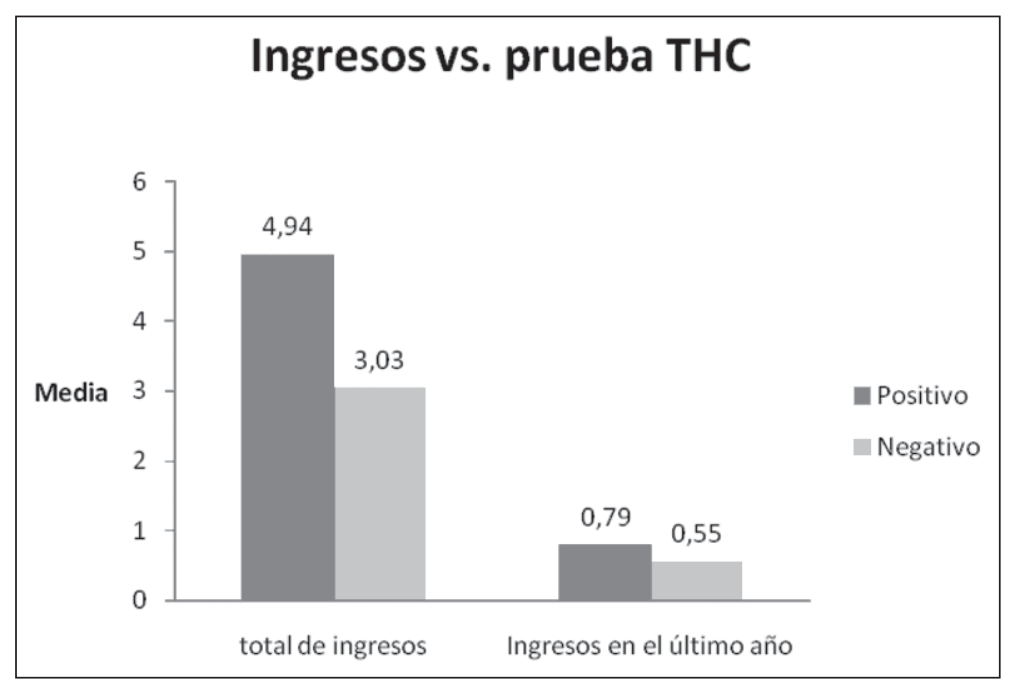

Figura 2. Ingresos a emergencia del hospital Vilardebó según prueba biológica positiva para THC. El consumo de THC tuvo una asociación significativa estadísticamente con mayor número de ingresos totales. la re-consulta de algunos pacientes. En total se realizaron 156 tests de orina. La causa de la diferencia se debió principalmente a la falta de colaboración del paciente y la sedación farmacológica que impedía el procedimiento.

La distribución por sexos fue de $62,2 \%$ para hombres y $37,8 \%$ para mujeres. El promedio de edades fue de 36,49 años, con un rango de 18 a 70. El grupo comprendido entre los 15 y 35 años representó un $42,4 \%$ del total.

El 72,6\% de los pacientes estudiados tuvo como diagnóstico Esquizofrenia (F20) y 18,2\% tuvieron como diagnóstico Trastornos Psicóticos Agudos y Transitorios (F23). Los motivos de ingresos más frecuentes fueron: síntomas psicóticos 46,6\%, conductas agresivas $28,4 \%$.

El $17,9 \%$ de la población testeada tuvo resultados positivos en orina para THC y/o COC, correspondiendo a 28 pacientes. En este grupo el $85,2 \%$ fueron hombres y $14,8 \%$ mujeres. La media de edad fue de 28,29 años $\pm 5,9$, con un mínimo de 18 y máximos de 47 años.

Para el grupo de hombres entre 15 a 35 años la prevalencia de testeado positivo fue de 33,9\%.

Del grupo de hombres con testeado positivo el $77,8 \%$ se encontraron en la franja etaria comprendida entre los 15 y 35 años.

El $70 \%$ de los pacientes con testeado positivo para THC lo admitieron en el autorreporte, en tanto el $50 \%$ de los que tuvieron testeado positivo para COC lo admitieron.

Los pacientes con testeado positivo para THC tuvieron una media de ingresos totales mayor comparado con los que no consumen $(4,94$ vs 3,03 $\mathrm{p}<0,03$ ). La diferencia en la media de ingresos en el último año no fue estadísticamente significativa. (Figura 2).

$\mathrm{Al}$ comparar los ingresos entre los pacientes con resultado positivo para COC y los no consumidores se halló una diferencia significativa tanto en la media de ingresos totales $(5,23$ vs $3,05 \mathrm{p}<$ $0,027)$ como del último año $(1,75$ vs $0,46 \mathrm{p}<0,00)$ (Figura 3).

Los motivos de ingresos más frecuentes en los pacientes con resultado positivo en orina para una o ambas sustancias fueron: síntomas psicóticos $42,9 \%$ y conductas agresivas $25 \%$. Con respecto a las conductas agresivas en los pacientes no se encontró una diferencia significativa entre los pacientes consumidores y no consumidores: $25 \%$ y $28,4 \%$ respectivamente.

\section{Discusión}

En la población testeada encontramos una prevalencia de 17,9\% de consumo reciente de THC 


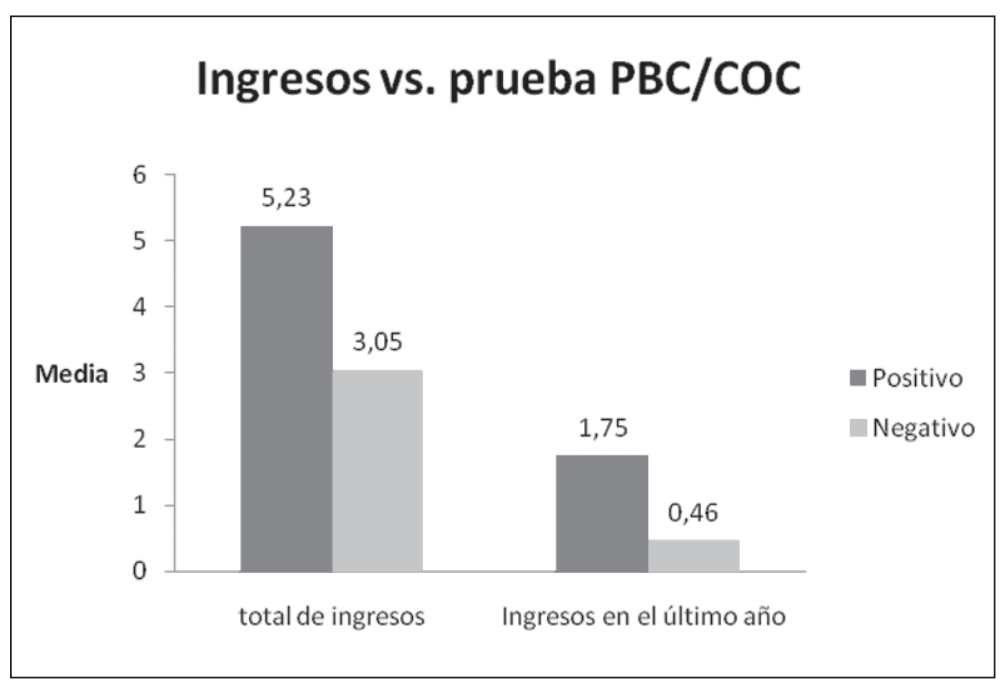

Figura 3. Ingresos en emergencia del hospital Vilardebó según prueba biológica positiva de $\mathrm{PBC} / \mathrm{COC}$. El consumo de PBC/COC tuvo una asociación significativa estadísticamente con mayor número de ingresos totales y también en el último año. y/o COC evidenciado a través de resultados positivos en orina. Otros estudios similares han obtenido resultados que oscilan entre 13\% (Cincinnati, E.E.U.U, 1995) ${ }^{6}, 21 \%$ (Dallas, E.E.U.U, 1993) ${ }^{7}$ y mayores del 30\% (Filadelfia, 1993, Brooklyn, $1993)^{8,9}$. Si bien existen diferencias metodológicas y diferencias socio-económicas-culturales con los estudios previamente mencionados, la similitud de los resultados nos lleva a adherirnos a la hipótesis de que los trastornos psicóticos tendrían una vulnerabilidad mayor para el consumo de sustancias psicoactivas $^{10}$.

La hipótesis anterior podría explicar el hecho que en el estudio realizado en la Emergencia del Hospital Pasteur (Hospital General) el porcentaje de tests positivos fue menos elevado para las mismas sustancias considerando el mismo grupo etario para pacientes de sexo masculino (15 a 35 años) siendo para THC 20,7 vs 13,9 y para COC/PBC 16,1 vs $11,4^{11}$ (Tabla 1). Esta vulnerabilidad para el consumo de sustancias en los pacientes psicóticos no estaría presente en los pacientes no psicóticos que consultan a la emergencia del Hospital General. Existen datos contradictorios en cuanto a la pertinencia de realizar de forma rutinaria screening en orina de sustancias en todo paciente que ingrese a la emergencia psiquiátrica, ya que se ha visto que en los pacientes sin trastornos psicóticos si bien la incidencia de consumo es alta, la fiabilidad del autorreporte también lo es ${ }^{12,13}$. Nuestro estudio apoya la pertinencia de realizar screening de sustancias psicoactivas fundamentalmente cocaína en los pacientes psicóticos, ya que la fiabilidad del autorreporte para cocaína fue del $50 \%$.

Desde el punto de vista de la población en Uruguay, los pacientes con trastornos psicóticos que ingresaron a la emergencia constituyen un grupo de elevado riesgo para el consumo si comparamos la prevalencia de consumo en los últimos 30 días según autorreporte entre los resultados de nuestro estudio y las cifras de la $4^{\text {a }}$ Encuesta Nacional de Hogares sobre Consumo de Drogas ${ }^{14}$ (Tabla 2). Dado que aproximadamente el $80 \%$ de los resultados positivos en orina se ubicó en los pacientes hombres entre 15 a 35 años, encontramos que el perfil de pacientes psicóticos consumidores es concordante con otros trabajos ${ }^{10,15}$.

Tabla 1. Comparación de testados positivos en hombres entre 15 y 35 años en Hospital General (Pasteur Marzo 2007) y Hospital Vilardebó (Octubre- Diciembre 2008)

\begin{tabular}{|lcc|}
\hline Sustancia & $\begin{array}{c}\text { Hospital } \\
\text { Vilardebó }\end{array}$ & $\begin{array}{c}\text { Hospital } \\
\text { Pasteur }\end{array}$ \\
\hline THC Positivo & $20,7 \%$ & $13,9 \%$ \\
\hline COC Positivo & $16,1 \%$ & $11,4 \%$ \\
\hline
\end{tabular}


Tabla 2. Prevalencia de consumo en los últimos 30 días según autorreporte. Datos de Hospital Vilardebó (Octubre- Diciembre 2008) comparado con datos de la $4^{\circ}$ Encuesta Nacional de Hogares (ENH) sobre consumo de drogas

\begin{tabular}{|lll|}
\hline Sustancia & Hospital Vilardebó & $\mathbf{4}^{\circ} \mathbf{E N H}$ \\
\hline THC & $17,1 \%$ & $3,3 \%$ \\
COC & $3,4 \%$ & $0,8 \%$ \\
\hline PBC & $8,9 \%$ & $0,1 \%$ \\
\hline
\end{tabular}

Los datos hallados en el presente estudio orientan hacia la hipótesis que el consumo de sustancias psicoactivas en este grupo favorecería la descompensación delirante, basándonos en que la mayoría de los pacientes con resultado positivo en el test de orina ingresaron por síntoma psicóticos.

De acuerdo la bibliografía citada en la introducción, el curso evolutivo de los pacientes con trastornos psicóticos que consumen drogas (marihuana, cocaína y pasta base de cocaína) parece empeorar cuando se lo compara con aquellos que no consumen ${ }^{16}$. Esto se evidencia por un incremento en el número de internaciones totales y del último año.

En lo referente al autorreporte el presente estudio tuvo índices de fiabilidad menores que aquellos obtenidos en Emergencia del Hospital General ${ }^{11}$. Esto podría deberse al hecho que son pacientes psicóticos descompensados, y por tanto su relato es poco fiable. Concordando con estudios anteriores en poblaciones similares, para el grupo de pacientes psicóticos el uso de tiras reactivas podría estar indicado ${ }^{9}$.

Con respecto a la asociación entre consumo de sustancias psicoactivas y la presencia de conductas agresivas, a diferencia de lo encontrado en el Hospital General donde las situaciones de violencia estaban sobrerrepresentadas en los pacientes consumidores ${ }^{11}$, en el presente estudio no encontramos diferencias estadísticamente significativas entre ambos grupos.

\section{Conclusiones}

La prevalencia del consumo reciente en los pacientes con Trastornos Psicótico que ingresaron a la emergencia de Hospital Vilardebó fue de 17,9\% evidenciado por prueba biológica. Esta cifra es más elevada comparada con estudios de emergencia del Hospital General y estudios sobre consumo a nivel de la población de nuestro país.

Los pacientes con testeado positivo mostraron una tasa total de ingresos mayor que aquella del grupo de los no consumidores, y los ingresos en el último año fueron claramente mayores para el grupo que consume cocaína y sus derivados.

Esto implicaría que para este grupo de pacientes sería necesario abordar no sólo el tratamiento de su psicosis sino también el del consumo de sustancias a la hora de mejorar su evolución.

La fiabilidad del autorreporte en el presente estudio fue menor que aquella encontrada en el estudio en la emergencia de Hospital General. Ello plantea la conveniencia de realizar screening de sustancias psicoactivas para los pacientes psicóticos que ingresan a sala de emergencia en particular si son hombres con edades comprendidas entre los 15 y 35 años.

\section{Agradecimientos}

A la Dirección del Hospital Vilardebó, al personal del Departamento de Emergencia y al Sistema de Información Hospitalaria (SIH) por su generosa colaboración para la realización de este estudio. A Laboratorios Celsius por la donación de las tiras reactivas.

A los médicos que también participaron en la tarea de campo: M. Ayala, S. Brescia, M. Bouissa, M. Cabral, E. Guglielmi, L. Montero, E. Olave, R. Pérez. 


\section{Resumen}

Se realizó un estudio descriptivo desde Octubre a Diciembre de 2008 en la Emergencia del Hospital Vilardebó estableciéndose la prevalencia del consumo de sustancias psicoactivas en los pacientes que ingresaban por Trastornos Psicóticos. La presencia de consumo reciente se estableció por autorreporte y test en orina para marihuana y cocaina/ pasta base de cocaina. La prevalencia del consumo reciente en los pacientes con Trastornos Psicóticos fue de 17,9\%. Los pacientes con testeado positivo mostraron una tasa total de ingresos mayor que aquella del grupo de los no consumidores, $y$ los ingresos en el último año fueron claramente mayores para el grupo que consume cocaina y sus derivados.

Palabras clave: Sustancias psicoactivas, psicosis, Emergencia de Hospital Psiquiátrico.

\section{Referencias}

1. Datos aportados por el Sistema de Información Hospitalaria (SIH) del Hospital Vilardebó.

2. San Molina L. Aspectos generales de la patología dual, en Consenso de la SEP sobre patología dual. $1^{\circ}$ ed. Barcelona: Editorial Arsmédica; 2004. Pp: 1.

3. Yaría, J. Introducción en Psicosis y drogadependencia. Buenos Aires: Editorial Gabas; 2008. pp 27-9.

4. Gregg L, Barrowclough C, Haddock G. Reasons for increased substance use in psychosis. Clin Psychol Rev 2007; 27 (4): 494-510.

5. Meikle M N, Urbanavicius J, Prunell G, Umpierrz E, Abín Carriquirry A, Scorza M. C. Primer estudio preclínico de la acción de la pasta base de cocaína en el sistema nervioso central. Rev Psiquiatría del Uruguay 2009; 73 (1): 25-36.

6. Strakowski S, Tohen M, Flaum M, et al. Substance abuse in psychotic disorders: associations with affective syndromes. Schizophrenia Research 1994; 14: 73-81.

7. Claase C A, Gilfillan S, Orsulak P, Carmody T J, Battaglia J, Rush A J. Substance use among patients with a psychotic disorder in a psychiatric emergency room. Psychiatric Serv 1997; 48 (3): 353-8.

8. Sanguineti V, Samuel S. Reported prevalence of drug abuse comorbidity in a citywide emergency rooms system. American Journal of Drug and
Alcohol Abuse 1993; 19: 443-50.

9. Elangovan N, Berman S, Meinzer A, Gianelli P, Miller H, Longmore W. Substance Abuse Among Patients Presenting at an Inner-City Psychiatric Emergency Room. Hosp Community Psychiatry 1993; 44: 782-4.

10. Margolese H C, Malchy L, Negrete J C, Tempier R, Gill K. Drug and alcohol use among patients with schizophrenia and related psychoses: levels and consequences. Schizophr Res 2004; 67 (2-3): 157-66. http://www.psiquiatria.com/articulos/ patologia_dual/45188/

11. Fielitz P, Suárez H, Bouissa M, Frontini M, López Rega G, Wschebor M, et al. Consumo de sustancias psicoactivas en pacientes de Emergencia del Hospital Pasteur. Revista de Psiquiatría del Uruguay. Rev Psiquiatr Urug 2009; 73 (2): 143-56. http://www.spu.org.uy/revista/dic2009/02_TO.pdf

12. Gilfillan S, Claasen C, Orsulak $\mathrm{P}$, et al. A comparison of psychotic and nonpsychotic substance users in the psychiatric emergency room. Psychiatric Services 1997; 49: 825-8.

13. Dhossche D, Rubinstein J. Drug detection in a suburban psychiatric emergency room. Annals of Clinical Psychiatry 1996; 8: 59-69.

14. Junta Nacional de Drogas (Presidencia de la República Oriental del Uruguay), ONUDD- OEA. $4^{\text {a }}$ Encuesta Nacional en Hogares sobre Consumo de Drogas. Edición Online. Montevideo. Noviembre 2006. http://www.infodrogas.gub.uy/html/ 
informesdocumentos/docs/presentación_4 ${ }^{\text {a_En-En- }}$ cuesta_Hogares_Drogas.pdf. Consultada Octubre 2009.

15. Míguez H, Magri R. Estudio nacional de hábitos tóxicos en el Uruguay. Acta Psiq Psicol Am Lat 1995; 41 (1): 13-23.
16. Schnell T, Neisius K, Daumann J, GouzoulisMayfrank E. Prevalence of psychosis/substance abuse comorbidity. Clinical-epidemiological findings from different treatment settings in a large German city. Nervenarzt 2010; 81 (3): 3238.

Correspondencia:

Pablo Fielitz G.

J. D. Jackson 881/301. Montevideo. CP 11200.

Uruguay.

Tel: (0598) 099692318.

E-mail:pfielitz@gmail.com 